\title{
Quark Transverse Parton Distribution at the Next-to-Next-to-Next-to-Leading Order
}

\author{
Ming-xing Luo, ${ }^{*}$ Tong-Zhi Yang $\odot,{ }^{\dagger}$ Hua Xing Zhu๑, ${ }^{\ddagger}$ and Yu Jiao Zhu ${ }^{\S}$ \\ Zhejiang Institute of Modern Physics, Department of Physics, Zhejiang University, Hangzhou 310027, China
}

(Received 17 December 2019; accepted 13 February 2020; published 5 March 2020)

\begin{abstract}
We report a calculation of the perturbative matching coefficients for the transverse-momentumdependent parton distribution functions for quark at the next-to-next-to-next-to-leading order in QCD, which involves calculation of nonstandard Feynman integrals with rapidity divergence. We introduce a set of generalized integration-by-parts equations, which allows an algorithmic evaluation of such integrals using the machinery of modern Feynman integral calculation.
\end{abstract}

DOI: 10.1103/PhysRevLett.124.092001

Introduction.-Transverse-momentum-dependent (TMD) parton distribution functions (PDFs) generalize the concept of PDFs by allowing the dependence on the intrinsic transverse momentum of struck parton, beside the conventional longitudinal momentum fraction. By probing the intrinsic confined transverse motion of parton inside the nucleon, TMD PDFs allow reconstruction of the 3D picture of the nucleon structure, as well as probing the parton orbital motion and the spin-orbit correlations between parton and nucleon. The possibility of precision measurement of TMD PDFs in both $p p$ and $e p$ scattering also probes fundamental aspects of QCD, such as gauge invariance and (non) universality. For these reasons, TMD PDFs play an increasingly central role in QCD theory and phenomenology in high energy collisions $[1,2]$. In light of the unprecedented precision of the future electron ion collider in TMD measurements [3], perturbative knowledge about TMD PDFs at the highest available order is desirable and is the main subject of this Letter.

The theoretical framework of TMD PDFs is substantially more complicated than the conventional PDFs, which is partly reflected by the existence of several different formulations of TMD factorization in the literature [1,4-14] (see Refs. [15,16] for a discussion of the different formulations). In this Letter, we adopt the rapidity renormalization group formalism of Refs. [14,17], which is based on soft-collinear effective theory (SCET) [18-22]. In this formalism, TMD factorization involves both TMD beam function $\mathcal{B}$ and TMD soft function $\mathcal{S}$. Schematically, for Drell-Yan production at low $q_{\perp}$,

$$
\frac{d \sigma}{d^{2} q_{\perp}} \sim \sigma_{0} H(Q) \int d^{2} b_{\perp} e^{i b_{\perp} \cdot q_{\perp}} \mathcal{B} \otimes \mathcal{B S}
$$

where $\sigma_{0}$ is the born cross section for the Drell-Yan process, $H(Q)$ is the quark electromagnetic form factor, and the convolution is in the longitudinal momentum fraction. The quark TMD beam function can be written as an operator matrix element in a hadron state with momenta $P$,

$$
\mathcal{B}_{q / N}\left(z, b_{\perp}\right)=\int \frac{d b_{-}}{4 \pi} e^{-i z b^{-} P^{+} / 2}\left\langle N(P)\left|\bar{\chi}_{n}\left(0, b^{-}, b_{\perp}\right) \frac{\not h}{2} \chi_{n}(0)\right| N(P)\right\rangle,
$$

where $\chi_{n}=W_{n}^{\dagger} \xi_{n}$ is the gauge invariant collinear quark field [23] in SCET, constructed from collinear quark field $\xi_{n}$ and path-ordered collinear Wilson line $W_{n}(x)=$ $\mathcal{P} \exp \left[\right.$ ig $\left.\int_{-\infty}^{0} d s \bar{n} \cdot A_{n}(x+\bar{n} s)\right]$ in some null direction $n=$ $\left(n^{+}, n^{-}, n_{\perp}\right)=\left(2,0,0_{\perp}\right)$ and $\bar{n}=\left(0,2,0_{\perp}\right) . b_{\perp}$ plays the

Published by the American Physical Society under the terms of the Creative Commons Attribution 4.0 International license. Further distribution of this work must maintain attribution to the author(s) and the published article's title, journal citation, and DOI. Funded by SCOAP ${ }^{3}$. role of a Fourier conjugate of the transverse momentum of the struck quark. The TMD soft function is a vacuum matrix element of time-ordered and anti-time-ordered soft Wilson lines,

$\mathcal{S}\left(b_{\perp}\right)=\frac{1}{N_{c}} \operatorname{Tr}\left\langle 0\left|\mathcal{T}\left[S_{\bar{n}}^{\dagger} S_{n}\left(0,0, b_{\perp}\right)\right] \overline{\mathcal{T}}\left[S_{n}^{\dagger} S_{\bar{n}}(0)\right]\right| 0\right\rangle$,

where $S_{n}(x)=\mathcal{P} \exp \left[i g \int_{-\infty}^{0} d s n \cdot A_{s}(x+s n)\right]$.

Similar to PDFs, the TMD beam function in Eq. (2) is intrinsically nonperturbative. At leading twist it admits a light-cone operator product expansion onto collinear PDFs, 


$$
\begin{aligned}
\mathcal{B}_{q / N}\left(z, b_{\perp}\right)= & \sum_{i} \int_{z}^{1} \frac{d \xi}{\xi} \mathcal{I}_{q i}\left(\xi, b_{\perp}\right) \phi_{i / N}(z / \xi) \\
& +\mathcal{O}\left(\left|b_{\perp}^{2}\right| \Lambda_{\mathrm{QCD}}^{2}\right)
\end{aligned}
$$

where, in principle, the matching coefficients $\mathcal{I}_{q i}$ are perturbatively calculable. The main complication is that the operator matrix element in Eqs. (2) and (3) are not well defined even within dimensional regularization. They suffer from the so-called rapidity divergence, which originates from gluon emission at infinite rapidity in the backward direction to the struck parton. Several regulators proposed to cure the rapidity divergence in TMD PDFs exist in the literature $[1,7,9,12,14,24-28]$, which allow one to calculate the matching coefficients in QCD to next-toleading order (NLO) $[1,7,9,12,14,29,30]$ and, relatively recently, to next-to-next-to-leading order (NNLO) [31-38]. These results provide a stringent test to the TMD factorization and facilitate some of the most precise theoretical predictions at the LHC [39-43].

In this Letter, we shall present for the first time a calculation of the matching coefficients for quark TMD beam function at the next-to-next-to-next-to-leading order $\left(\mathrm{N}^{3} \mathrm{LO}\right)$ within the exponential regularization scheme [27] in a completely analytic form. We also provide results for the regulator-independent TMD PDFs, by combining the beam function with the $\mathrm{N}^{3} \mathrm{LO}$ TMD soft function [44]. Our calculation contains all the color structures and all the partonic channels and represents a major step toward precision TMD physics. To facilitate this calculation, we introduce a set of generalized integration-by-parts equations that allows an algorithmic evaluation of rapidity divergent integrals, using the powerful machinery of modern Feynman diagram calculation, which shall be explained in the next section.

The method.-Since the matching coefficients can be calculated in short-distance perturbation theory, we can use asymptotic quark and gluon states instead of the hadron state $N(P)$ in Eq. (2). Furthermore, we can insert a complete state of $|X\rangle\langle X|$, which also consists of asymptotic quark and gluon states, between $\bar{\chi}_{n}$ and $\chi_{n}$. This converts the calculation of the perturbative TMD beam function to the calculation of the semi-inclusive partonic cross section,

$$
\mathcal{B}_{q / i}\left(z, b_{\perp}\right)=\sum_{L=0} \sum_{n+m=L} \mathcal{B}_{q / i}^{(n, m)}\left(z, b_{\perp}\right),
$$

where $\mathcal{B}_{q / i}^{(n, m)}\left(z, b_{\perp}\right)$ represents an $n$-loop, $m$-real emission contribution for the semi-inclusive subprocess $i \rightarrow q+X$ at $\mathcal{O}\left(\alpha_{s}^{n+m}\right)$,

$$
\mathcal{B}_{q / i}^{(n, m)}\left(z, b_{\perp}\right)=\int \frac{d^{d-2} \tilde{K}_{\perp}}{\left|\tilde{K}_{\perp}^{2}\right|^{-\epsilon}} e^{-i b_{\perp} \cdot \tilde{K}_{\perp}} \tilde{\mathcal{B}}_{q / i}^{(n, m)}\left(z, \tilde{K}_{\perp}\right),
$$

with $d=4-2 \epsilon$ the space-time dimension. We have suppressed the renormalization scale $\mu$ and rapidity scale $\nu$ in the argument for simplicity. The function $\tilde{\mathcal{B}}_{q / i}^{(n, m)}\left(z, \tilde{K}_{\perp}\right)$ has simple power and logarithmic dependence on $\tilde{K}_{\perp}$, which can be integrated easily $[36,37]$. The function $\tilde{\mathcal{B}}_{q / i}^{(n, m)}\left(z, \tilde{K}_{\perp}\right)$ can be calculated as

$$
\begin{aligned}
\tilde{\mathcal{B}}_{q / i}^{(n, m)}\left(z, \tilde{K}_{\perp}\right)= & {\left[\lim _{\tau \rightarrow 0} \frac{2 \int d^{d} K}{V_{d-2}} e^{-b_{0} \tau_{P}^{P \cdot K}} \delta\left[K^{+}-P^{+}(1-z)\right] \times \delta\left(\tilde{K}_{\perp}^{2}-K_{\perp}^{2}\right) \mu^{2 \epsilon(n+m)} \prod_{j=0}^{n} \frac{\int d^{d} l_{j}}{(2 \pi)^{d}} \prod_{r=0}^{m} \frac{\int d^{d} k_{r}}{(2 \pi)^{d-1}} \delta_{+}\left(k_{r}^{2}\right)\right.} \\
& \left.\times \delta^{(d)}\left(K-\sum_{r=1}^{m} k_{r}\right)\left|\overline{\operatorname{Sp}}_{q \leftarrow i}(P,\{l\},\{k\})\right|^{2}\right]\left.\right|_{\tau \rightarrow 1 / \nu},
\end{aligned}
$$

where $e^{-b_{0} \tau\left(P \cdot K / P^{+}\right)}$is the exponential regulator [27] in the collinear sector [36]. $\left|\overline{\operatorname{Sp}}_{q \leftarrow i}(P,\{l\},\{k\})\right|^{2}$ is the spin and color averaged squared splitting amplitudes for $i \rightarrow q+X$ with $n$ loops (counting at the squared amplitude level) and $m$ real emission. $K^{\mu}$ is the total four momentum of $X . b_{0}=$ $2 e^{-2 \gamma_{E}}$ is a conventional factor, and $V_{d}=2 \pi^{d / 2} / \Gamma(d / 2)$ is the volume of $d$ sphere. It is easy to see from Eq. (7) that the dependence on $\tilde{K}_{\perp}$ only enters through $\ln \left(\left|\tilde{K}_{\perp}^{2}\right| / \mu^{2}\right)$ or $\ln \left(\left|\tilde{K}_{\perp}^{2}\right| / \nu^{2}\right)$. Note that $K_{\perp}^{2}=K^{2}-K^{-} K^{+}=K^{2}-$ $K^{-} P^{+}(1-z)$. Using reverse unitarity [45], the delta functions in Eq. (7) can be considered as "cut" propagators. Therefore, $\tilde{\mathcal{B}}_{q / i}^{(n, m)}\left(z, \tilde{K}_{\perp}\right)$ can be regarded as an $(n+m)$ loop Feynman integral in the algebraic sense, if not for the exponential regulator. The main new idea of this Letter is the introduction of a set of generalized integration-by-parts (IBPs) equation [46,47], which takes into account also the exponential regularization factor in Eq. (7).

Specifically, the generalized IBPs equations can be written as

$$
\begin{aligned}
0 & =\int d^{d} q \frac{\partial}{\partial q^{\mu}}\left[e^{-b_{0} \tau \frac{P \cdot K}{P^{+}}} F(\{\tilde{l}\})\right] \\
& = \begin{cases}\int d^{d} q e^{-b_{0} \tau \frac{P \cdot K}{P^{+}}}\left[-b_{0} \tau \frac{P_{\mu}}{P^{+}}+\frac{\partial}{\partial q^{\mu}}\right] F(\{\tilde{l}\}), & q=K, \\
\int d^{d} q e^{-b_{0} \tau \cdot \frac{P \cdot K}{P^{+}}} \frac{\partial}{\partial q^{\mu}} F(\{\tilde{l}\}), & q \neq K,\end{cases}
\end{aligned}
$$

where $F(\{\tilde{l}\})$ is some Feynman integrand, and $\{\tilde{l}\}=$ $\left\{l_{1}, l_{2}, \ldots, l_{n}, k_{1}, k_{2}, \ldots, k_{m-1}, K\right\}$ is the set of integration 


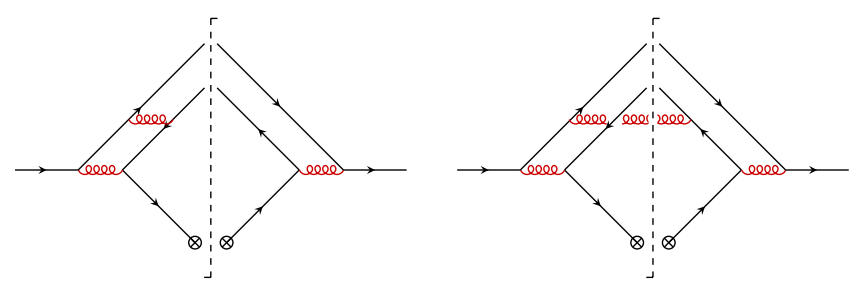

FIG. 1. Representative cut diagram for the color structure $d^{A B C} d_{A B C}=\left(N_{c}^{2}-1\right)\left(N_{c}^{2}-4\right) / N_{c}$ from VRR (left) and RRR (right).

momenta. Note that we have used the delta function in Eq. (7) to kill one of the integration momenta $k_{m}$. We generate the required IBPs equations in this way using inhouse Mathematica code and LITERED [48]. The obtained IBPs equations and the integrals are exported to FIRE [49], where all the integrals can be reduced to a set of master integrals (MIs).

The main task in this Letter is to calculate Eq. (7) at three loops, i.e., $m+n=3$. The contribution at this order can be classified by the number of loops and the number of real emission: triple-real (RRR), double-real single-virtual (VRR), double-virtual single-real (VVR), and the square of real-virtual $\left[(\mathrm{VR})^{2}\right]$. For the VVR part, we use the results in Ref. [50], analytically continue them to a spacelike case, and directly integrate over the single particle phase space. The $(\mathrm{VR})^{2}$ part is relatively simple. we use the method presented in Ref. [36] to handle this part.

The main challenge of this calculation is the VRR and RRR parts. Representative cut Feynman diagrams are shown in Fig. 1. We generate the integrand for VRR and RRR in QGRAF [51], with color-Dirac algebra and integrand manipulation aided by FORM [52], FEYNCALC [53], and APART [54]. Using the above mentioned new idea, we then pass the integrand and the generalized IBPs equations to FIRE for integral reduction. After exploring symmetry between different integral families, we find about 900 MIs for VRR and RRR in total. To solve these MIs, we use the method of differential equations (DEs) [55-57].

Note that up until this stage we have kept the rapidity regulator $\tau$ finite. The resulting MIs are functions of $z$ and $\tau$, and DEs in $z$ and $\tau$ can be constructed in the standard way. The next step is to take the $\tau \rightarrow 0$ limit, as in Eq. (7). We do so by expanding the DEs and the MIs around $\tau=0$ consistently, but keeping the full functional dependence on $z$. We write a MI $f_{i}$ as a double series in $\tau$ and $\ln \tau$,

$$
f_{i}(z, \tau, \epsilon) \stackrel{\tau \rightarrow 0}{=} \sum_{j} \sum_{k=0} f_{i}^{(j, k)}(z, \epsilon) \tau^{j} \ln ^{k} \tau .
$$

Note that for an individual diagram in the Feynman gauge, the result is absent of powerlike singularity in $\tau$. However, application of the generalized IBPs leads to power divergence for an individual MI. We found by observation that only $j \geq-1$ is needed in Eq. (9), by checking explicitly that terms with $j=-2$ and $j=-3$ vanish. In principle, we can also have terms like $\tau^{\epsilon}$ in the expansion. However, such terms can be discarded, because we can always analytically continue to a region where $\epsilon>0$, and take $\tau \rightarrow 0$, and then take $\epsilon \rightarrow 0$. In practice, we have checked this by enlarging the ansatz in Eq. (9) by multiplying with $\tau^{ \pm n \epsilon}$ for $n=0, \ldots, 4$. We found that, when substituting the MIs into the integrand, the coefficients for $n=1, \ldots, 4$ always vanish for each diagram. Therefore, these terms are spurious at the diagram level, and we discard them in Eq. (9) to simplify our calculation. We then substitute the double series in Eq. (9) into the system of DEs, which are expanded in $\tau$, but with full $z$ dependence. By equating the $\tau^{j} \ln ^{k} \tau$ coefficient in the DEs, we obtain a closed system of DEs in $z$ for $f_{i}^{(j, k)}$. By considering the double series expansion, the number of MIs are reduced to about 500 in total for VRR and RRR.

The system of DEs in $z$ can now be solved by the standard approach. They are most conveniently solved by converting into the canonical form [57] by proper choice of MIs [58-60]. For individual VRR or RRR, the alphabet consists of five letters,

$$
\left\{z, 1-z, 1+z, 2-z, z^{2}-z+1\right\} .
$$

The DEs can be solved order-by-order in $\epsilon$ easily in terms of Goncharov polylogarithms. Remarkably, after summing the VRR and RRR contributions, and substituting in the boundary constants determined below, the latter two letters drop out from the sum. Therefore, harmonic polylogarithms (HPLs) [61] are sufficient to describe the final results.

To determine the boundary constants for the DEs, we consider the threshold limit of the MIs, $z \rightarrow 1$. Following Ref. [36], we define the so-called fully differential beam function [62],

$$
\hat{\mathcal{B}}_{q / i}^{(n, m)}\left(z, K^{+}, K^{-}, K_{\perp}\right)=\prod_{j=0}^{n} \frac{\int d^{d} l_{j}}{(2 \pi)^{d}} \prod_{r=0}^{m} \frac{\int d^{d} k_{r}}{(2 \pi)^{d-1}} \delta_{+}\left(k_{r}^{2}\right) \times \delta^{(d)}\left(K-\sum_{r=1}^{m} k_{r}\right) \mu^{2 \epsilon(n+m)}\left|\overline{\operatorname{Sp}}_{q \leftarrow i}(P,\{l\},\{k\})\right|^{2} .
$$

The original $\tilde{\mathcal{B}}$ is simply obtained by integrating the $K^{-}$component,

$$
\tilde{\mathcal{B}}_{q / i}^{(n, m)}\left(z, \tilde{K}_{\perp}\right)=\left.\left(\lim _{\tau \rightarrow 0} \frac{\left|\tilde{K}_{\perp}^{2}\right|^{-\epsilon}}{V_{d-2}} \int_{0}^{\infty} d K^{-} e^{-b_{0} \tau_{\frac{P}{P^{+}}}} \cdot \hat{\mathcal{B}}_{q / i}^{(n, m)}\left(z, P^{+}(1-z), K^{-}, \tilde{K}_{\perp}\right)\right)\right|_{\tau \rightarrow 1 / \nu}
$$


The advantage of having the fully differential function is that now the threshold limit can be taken at the integrand level. For that purpose, we adopt the strategy of expansion by region [63]. For RRR, $z \rightarrow 1$ forces the leading region to be $K^{\mu} \rightarrow 0$. VRR is more complicated. In addition to $K^{\mu} \rightarrow 0$, we also need to consider the scaling in the loop momentum, which can be either soft or collinear [64]. Ultimately, expansion by region relates all the boundary constants to those computed for soft-virtual corrections to Higgs production at $\mathrm{N}^{3} \mathrm{LO}$. We have performed an independent calculation for these constants and found agreement with those in the literature [64-68].
The results. - We are ready to combine all the ingredients and present the final results. The bare TMD beam function computed in the last section contains poles in $\epsilon$ up to $1 / \epsilon^{6}$. Using the known renormalization constant and the PDF counterterms (which contain the famous three-loop splitting kernel $[69,70])$, we find that all the poles cancel, and finite matching coefficients can be extracted. This provides a stringent check to our calculation. For the convenience of the reader, the relevant renormalization counterterms are collected in the Supplemental Material [71]. We refer to Refs. [36,37] for the detailed renormalization procedure.

The finite matching coefficient obeys the renormalization group equation,

$$
\begin{aligned}
\frac{d}{d \ln \mu} \mathcal{I}_{q i}\left(z, b_{\perp}, P^{+}, \mu, \nu\right)= & 2\left(\Gamma^{\mathrm{cusp}}\left[\alpha_{s}(\mu)\right] \ln \frac{\nu}{z P^{+}}+\gamma^{B}\left[\alpha_{s}(\mu)\right]\right) \mathcal{I}_{q i}\left(z, b_{\perp}, P^{+}, \mu, \nu\right) \\
& -2 \sum_{j} \mathcal{I}_{q j}\left(z, b_{\perp}, P^{+}, \mu, \nu\right) \otimes P_{j i}\left(z, \alpha_{s}(\mu)\right),
\end{aligned}
$$

where the anomalous dimension can be found in the Supplemental Material [71]. It also obeys the rapidity evolution equation [14],

$$
\frac{d}{d \ln \nu} \mathcal{I}_{q i}\left(z, b_{\perp}, P^{+}, \mu, \nu\right)=-2\left(\int_{\mu}^{b_{0} /\left|b_{\perp}\right|} \frac{d \bar{\mu}}{\bar{\mu}} \Gamma^{\operatorname{cusp}}\left[\alpha_{s}(\bar{\mu})\right]+\gamma^{R}\left[\alpha_{s}\left(b_{0} /\left|b_{\perp}\right|\right)\right]\right) \mathcal{I}_{q i}\left(z, b_{\perp}, P^{+}, \mu, \nu\right) .
$$

The new results of this Letter are the initial conditions (coefficient functions) for these equations $I_{i j}(z)=$ $\mathcal{I}_{i j}\left(z, b_{\perp}, P^{+}, \mu=b_{0} /\left|b_{\perp}\right|, \nu=z P^{+}\right)$. As mentioned before, the three-loop results can be written solely in terms of HPLs, which we believe is quite remarkable. The full results can be found, along with computer readable files, in the Supplemental Material [71]. The TMD beam function depends on the rapidity regulator being used. Rapidityregulator-independent TMD PDFs can be simply obtained using [36,37]

$$
f_{T, i j}\left(z, b_{\perp}, P^{+}, \mu\right)=\mathcal{I}_{i j}\left(z, b_{\perp}, P^{+}, \mu, \nu\right) \sqrt{\mathcal{S}\left(b_{\perp}, \mu, \nu\right)} .
$$

The TMD soft function $\mathcal{S}\left(b_{\perp}, \mu, \nu\right)$ is also known to $\mathrm{N}^{3} \mathrm{LO}$ [44]. Therefore, quark TMD PDFs can also be extracted to this order, see the Supplemental Material [71].

It is interesting to consider the asymptotic limit of the coefficient functions. In the threshold limit, we find the nonvanishing component to be

$$
\begin{aligned}
I_{q q}^{(2)}= & \frac{1}{(1-z)_{+}}\left[\left(28 \zeta_{3}-\frac{808}{27}\right) C_{A} C_{F}+\frac{224}{27} C_{F} N_{f} T_{F}\right], \\
I_{q q}^{(3)}= & \frac{1}{(1-z)_{+}}\left[\left(-\frac{128 \zeta_{3}}{9}-\frac{7424}{729}\right) C_{F} N_{f}^{2} T_{F}^{2}+\left(-\frac{1648 \zeta_{2}}{81}-\frac{1808 \zeta_{3}}{27}+\frac{40 \zeta_{4}}{3}+\frac{125252}{729}\right) C_{A} C_{F} N_{f} T_{F}\right. \\
& \left.+\left(-\frac{176}{3} \zeta_{3} \zeta_{2}+\frac{6392 \zeta_{2}}{81}+\frac{12328 \zeta_{3}}{27}+\frac{154 \zeta_{4}}{3}-192 \zeta_{5}-\frac{297029}{729}\right) C_{A}^{2} C_{F}+\left(-\frac{608 \zeta_{3}}{9}-32 \zeta_{4}+\frac{3422}{27}\right) C_{F}^{2} N_{f} T_{F}\right],
\end{aligned}
$$

where $I_{i j}^{(n)}$ is the expansion coefficient of $\left[\alpha_{s} /(4 \pi)\right]^{n}$. One can also identify the coefficient with the rapidity anomalous dimension

$$
I_{q q}^{(2)}(z)=\frac{2 \gamma_{1}^{R}}{(1-z)_{+}}, \quad I_{q q}^{(3)}(z)=\frac{2 \gamma_{2}^{R}}{(1-z)_{+}},
$$

where $\gamma_{1(2)}^{R}$ are the two(three)-loop rapidity anomalous dimension $[44,79]$. This result was conjectured in Ref. [35] and was understood using joint $q_{T}$ and threshold resummation [80] (see also Ref. [81]). This provides another check to our calculation. 
We can also consider the high energy limit $z \rightarrow 0$, which is closely related to small- $x$ physics. The leading terms are

$$
\begin{aligned}
I_{q g}^{(2)}= & \frac{2 C_{A} T_{F}}{z}\left(\frac{172}{27}-\frac{8 \zeta_{2}}{3}\right), \\
I_{q q}^{(2)}=I_{q q^{\prime}}^{(2)}=I_{q \bar{q}}^{(2)}=I_{q \bar{q}^{\prime}}^{(2)}= & \frac{2 C_{F} T_{F}}{z}\left(\frac{172}{27}-\frac{8 \zeta_{2}}{3}\right), \\
I_{q g}^{(3)}= & \frac{2 T_{F}}{z}\left[\left(\frac{208 \zeta_{2}}{9}+\frac{32 \zeta_{3}}{3}-\frac{17152}{243}\right) C_{A}^{2} \ln z+\left(\frac{160 \zeta_{2}}{27}-\frac{32 \zeta_{3}}{9}-\frac{3164}{729}\right) C_{A} N_{f} T_{F}\right. \\
& +\left(-16 \zeta_{2}+\frac{512 \zeta_{3}}{9}+\frac{32 \zeta_{4}}{3}-\frac{269}{9}\right) C_{A} C_{F}+\left(\frac{12536 \zeta_{2}}{81}+\frac{1096 \zeta_{3}}{9}+\frac{920 \zeta_{4}}{9}-\frac{470494}{729}\right) C_{A}^{2} \\
& \left.+\left(-\frac{512 \zeta_{2}}{27}-\frac{64 \zeta_{3}}{9}+\frac{40184}{729}\right) C_{F} N_{f} T_{F}\right], \\
I_{q q}^{(3)}=I_{q q^{\prime}}^{(3)}=I_{q \bar{q}}^{(3)}=I_{q \bar{q}^{\prime}}^{(3)}= & \frac{2 T_{F}}{z}\left[\left(\frac{208 \zeta_{2}}{9}+\frac{32 \zeta_{3}}{3}-\frac{17152}{243}\right) C_{A} C_{F} \ln z+\left(\frac{12008 \zeta_{2}}{81}+120 \zeta_{3}+\frac{920 \zeta_{4}}{9}-\frac{456266}{729}\right) C_{A} C_{F}\right. \\
& \left.+\left(-\frac{32 \zeta_{2}}{9}-\frac{64 \zeta_{3}}{9}+\frac{16928}{729}\right) C_{F} N_{f} T_{F}+\left(-16 \zeta_{2}+\frac{512}{9} \zeta_{3}+\frac{32}{3} \zeta_{4}-\frac{269}{9}\right) C_{F}^{2}\right] .
\end{aligned}
$$

There has been recent progress in understanding TMD PDFs at small $x$ [82-85]. Our explicit results provide useful data through next-to-leading logarithmic accuracy, which can foster further progress.

To estimate the size of the three-loop corrections, we plot the ratio of $\mathrm{N}^{3} \mathrm{LO}$ and NNLO coefficient functions for $0<z<1$ in Fig. 2,

$$
\frac{\mathrm{N}^{3} \mathrm{LO}}{\mathrm{NNLO}}=1+\frac{\left(\frac{\alpha_{s}}{4 \pi}\right)^{3} I_{i j}^{(3)}(z)}{\frac{\alpha_{s}}{4 \pi} I_{i j}^{(1)}(z)+\left(\frac{\alpha_{s}}{4 \pi}\right)^{2} I_{i j}^{(2)}(z)} .
$$

It can be seen that the three-loop corrections are nonnegligible and have nontrivial shape dependence. Note that the coefficient functions are not ordinary functions of $z$, but distributions. To see the perturbative convergence of the TMD PDFs, we define an integrated version of it,

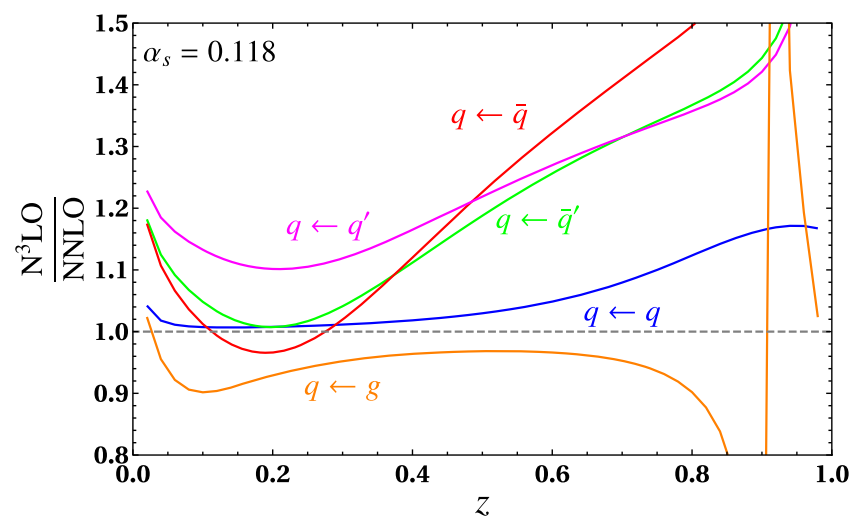

FIG. 2. $K$ factor at $\mathrm{N}^{3} \mathrm{LO}$ for the coefficient functions. Numerical evaluation of HPLs are made with the HPL package [86].

$$
B_{q / N}\left(z, q_{T}^{\max }\right)=\sum_{i} \int_{0}^{q_{T}^{\max }} d q_{T} \int_{z}^{1} \frac{d \xi}{\xi} f_{T, q i}\left(\xi, q_{T}\right) \phi_{i / N}(z / \xi),
$$

where $f_{T, q i}\left(\xi, q_{T}\right)$ is the momentum-space version of the TMD coefficients defined in Eq. (15), and $q_{T}^{\max }$ is a UV cutoff, below which the TMD approximation can be justified. In Fig. 3 we depict $z B_{u / N}$ with $q_{T}^{\max }=10 \mathrm{GeV}$ at various perturbative orders, where $N$ is the proton. It can be seen that, for moderate and small $z$, NLO and NNLO corrections are large and there is no obvious perturbative convergence. On the other hand, after inclusion of the $\mathrm{N}^{3} \mathrm{LO}$ corrections, the perturbative series is stabilized even for $z$ as small as $10^{-4}$. This gives us confidence that

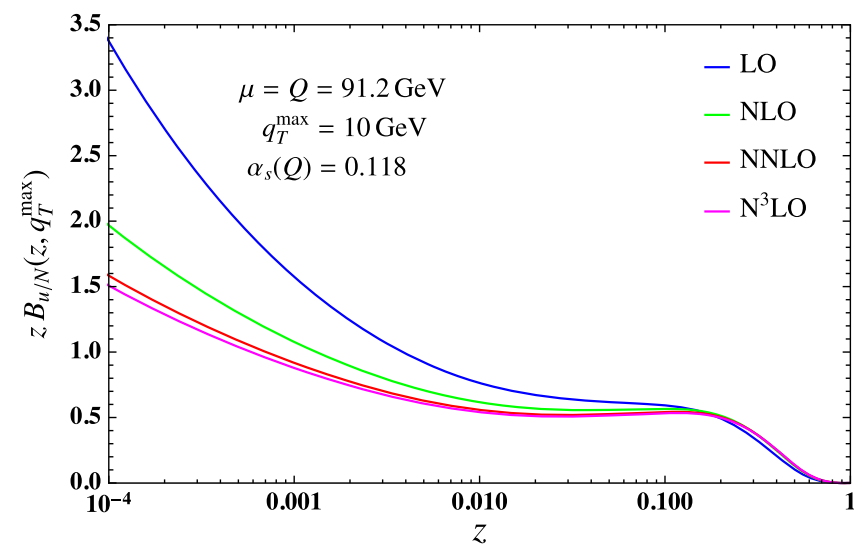

FIG. 3. Integrated TMD PDFs at various perturbative order. We use NNPDF30_nnlo_as_0118 [87] throughout the numerical calculation. 
perturbative uncertainties are under good control at this order.

Discussion.-We have calculated for the first time the quark TMD beam function and TMD PDFs at the $\mathrm{N}^{3} \mathrm{LO}$ in QCD. The key new idea is the generalization of the IBPs equations to Feynman integrals with the exponential regulator for rapidity divergence. We hope that the $\mathrm{N}^{3} \mathrm{LO}$ results can improve our understanding about TMD factorization. There are several interesting directions to pursue in the future.

An obvious next thing to calculate is the gluon TMD PDFs at $\mathrm{N}^{3}$ LO. Past experience [37] shows that gluon TMD PDFs share the same set of MIs with the quark TMD PDFs, which significantly simplifies the calculation. It would also be interesting to calculate the TMD fragmentation function at $\mathrm{N}^{3}$ LO. At NNLO, the timelike TMD functions can be obtained from spacelike ones by analytic continuation [3537]. However, it is known from the splitting function calculation that naive analytic continuation must be supplemented with physical constraint from reciprocity consideration [88], and still, small uncertainty remains [8991]. It would be interesting to see if a direct calculation of timelike TMD functions can shed light on this problem. Our results represent the last missing ingredient for generalizing $q_{T}$ subtraction [92] to $\mathrm{N}^{3} \mathrm{LO}$ (see also Refs. [41,81]). We note that there has also been progress in calculating the beam function for beam thrust [93], where the leading color contribution at $\mathrm{N}^{3} \mathrm{LO}$ has become available very recently [94]. However, to achieve $\mathrm{N}^{3} \mathrm{LO}$ accuracy based on $N$ jettiness subtraction $[95,96]$, the $\mathrm{N}^{3} \mathrm{LO}$ (beam) thrust soft function is still missing $[97,98]$. Once a fully differential $\mathrm{N}^{3} \mathrm{LO}$ prediction with $q_{T}$ subtraction becomes available, it would also be important to consider the perturbative power corrections, where rapidity logarithms beyond leading power need to be understood better [28,99] (see also $[100,101])$. It would also be interesting to study if the idea of generalized IBPs introduced in this Letter, perhaps with some modification, is helpful in problems where nonstandard Feynman propagators are encountered, such as two-loop $q_{T}$ soft function for top-quark pair production [102,103] or thrust and hemisphere soft function $[97,98,104]$ with a step function in the integrand. Integrals with the latter form are particularly interesting since they appear frequently in precision jet substructure [105]. Finally, given the success of the exponential regulator in perturbative calculations, it would be interesting to see if it can be applied to TMD PDFs on lattice, where rapid progress is being made recently [106-110].

We thank V. Shtabovenko for valuable discussions and C. Duhr, T. Gehrmann, and M. Jaquier for providing us with the results of Ref. [50] in electronic form. This work was supported in part by National Natural Science Foundation of China under Grants No. 11975200 and No. 11935013 and the Zhejiang University Fundamental Research Funds for the Central Universities (2017QNA3007, 2019QNA3005). *mingxingluo@zju.edu.cn

†yangtz@zju.edu.cn

¥zhuhx@zju.edu.cn

§huyujiao@zju.edu.cn

[1] J. Collins, Foundations of Perturbative QCD (Cambridge University Press, Cambridge, 2011).

[2] R. Angeles-Martinez et al., Acta Phys. Pol. B 46, 2501 (2015).

[3] A. Accardi et al., Eur. Phys. J. A 52, 268 (2016).

[4] Y. L. Dokshitzer, D. Diakonov, and S. I. Troian, Phys. Lett. 79B, 269 (1978).

[5] G. Parisi and R. Petronzio, Nucl. Phys. B154, 427 (1979).

[6] J. C. Collins and D. E. Soper, Nucl. Phys. B194, 445 (1982).

[7] J. C. Collins, D. E. Soper, and G. F. Sterman, Nucl. Phys. B250, 199 (1985).

[8] X.-d. Ji, J.-P. Ma, and F. Yuan, Phys. Lett. B 597, 299 (2004).

[9] X.-d. Ji, J.-p. Ma, and F. Yuan, Phys. Rev. D 71, 034005 (2005).

[10] G. Bozzi, S. Catani, D. de Florian, and M. Grazzini, Nucl. Phys. B737, 73 (2006).

[11] I. O. Cherednikov and N. G. Stefanis, Phys. Rev. D 77, 094001 (2008).

[12] T. Becher and M. Neubert, Eur. Phys. J. C 71, 1665 (2011).

[13] M. G. Echevarria, A. Idilbi, A. Schäfer, and I. Scimemi, Eur. Phys. J. C 73, 2636 (2013).

[14] J.-Y. Chiu, A. Jain, D. Neill, and I. Z. Rothstein, J. High Energy Phys. 05 (2012) 084.

[15] J. C. Collins and T. C. Rogers, Phys. Rev. D 87, 034018 (2013).

[16] J. Collins and T. C. Rogers, Phys. Rev. D 96, 054011 (2017).

[17] J.-y. Chiu, A. Jain, D. Neill, and I. Z. Rothstein, Phys. Rev. Lett. 108, 151601 (2012).

[18] C. W. Bauer, S. Fleming, and M. E. Luke, Phys. Rev. D 63, 014006 (2000).

[19] C. W. Bauer, S. Fleming, D. Pirjol, and I. W. Stewart, Phys. Rev. D 63, 114020 (2001).

[20] C. W. Bauer, D. Pirjol, and I. W. Stewart, Phys. Rev. D 65, 054022 (2002).

[21] C. W. Bauer, S. Fleming, D. Pirjol, I. Z. Rothstein, and I. W. Stewart, Phys. Rev. D 66, 014017 (2002).

[22] M. Beneke, A. P. Chapovsky, M. Diehl, and T. Feldmann, Nucl. Phys. B643, 431 (2002).

[23] C. W. Bauer and I. W. Stewart, Phys. Lett. B 516, 134 (2001).

[24] T. Becher and G. Bell, Phys. Lett. B 713, 41 (2012).

[25] J.-y. Chiu, A. Fuhrer, A. H. Hoang, R. Kelley, and A. V. Manohar, Phys. Rev. D 79, 053007 (2009).

[26] M. G. Echevarria, I. Scimemi, and A. Vladimirov, Phys. Rev. D 93, 054004 (2016).

[27] Y. Li, D. Neill, and H. X. Zhu, arXiv:1604.00392.

[28] M. A. Ebert, I. Moult, I. W. Stewart, F. J. Tackmann, G. Vita, and H. X. Zhu, J. High Energy Phys. 04 (2019) 123.

[29] S. M. Aybat and T. C. Rogers, Phys. Rev. D 83, 114042 (2011).

[30] M. G. Echevarria, A. Idilbi, and I. Scimemi, J. High Energy Phys. 07 (2012) 002. 
[31] S. Catani and M. Grazzini, Eur. Phys. J. C 72, 2013 (2012); 72, 2132(E) (2012).

[32] S. Catani, L. Cieri, D. de Florian, G. Ferrera, and M. Grazzini, Eur. Phys. J. C 72, 2195 (2012).

[33] T. Gehrmann, T. Lubbert, and L. L. Yang, Phys. Rev. Lett. 109, 242003 (2012).

[34] T. Gehrmann, T. Luebbert, and L. L. Yang, J. High Energy Phys. 06 (2014) 155.

[35] M. G. Echevarria, I. Scimemi, and A. Vladimirov, J. High Energy Phys. 09 (2016) 004.

[36] M.-X. Luo, X. Wang, X. Xu, L. L. Yang, T.-Z. Yang, and H. X. Zhu, J. High Energy Phys. 10 (2019) 083.

[37] M.-X. Luo, T.-Z. Yang, H. X. Zhu, and Y. J. Zhu, J. High Energy Phys. 01 (2020) 040.

[38] D. Gutierrez-Reyes, S. Leal-Gomez, I. Scimemi, and A. Vladimirov, J. High Energy Phys. 11 (2019) 121.

[39] X. Chen, T. Gehrmann, E. W. N. Glover, A. Huss, Y. Li, D. Neill, M. Schulze, I. W. Stewart, and H. X. Zhu, Phys. Lett. B 788, 425 (2019).

[40] W. Bizoń, X. Chen, A. Gehrmann-De Ridder, T. Gehrmann, N. Glover, A. Huss, P. F. Monni, E. Re, L. Rottoli, and P. Torrielli, J. High Energy Phys. 12 (2018) 132.

[41] L. Cieri, X. Chen, T. Gehrmann, E. W. N. Glover, and A. Huss, J. High Energy Phys. 02 (2019) 096.

[42] W. Bizon, A. Gehrmann-De Ridder, T. Gehrmann, N. Glover, A. Huss, P. F. Monni, E. Re, L. Rottoli, and D. M. Walker, Eur. Phys. J. C 79, 868 (2019).

[43] V. Bertone, I. Scimemi, and A. Vladimirov, J. High Energy Phys. 06 (2019) 028.

[44] Y. Li and H. X. Zhu, Phys. Rev. Lett. 118, 022004 (2017).

[45] C. Anastasiou and K. Melnikov, Nucl. Phys. B646, 220 (2002).

[46] K. G. Chetyrkin and F. V. Tkachov, Nucl. Phys. B192, 159 (1981).

[47] S. Laporta, Int. J. Mod. Phys. A 15, 5087 (2000).

[48] R. N. Lee, arXiv:1212.2685.

[49] A. V. Smirnov, Comput. Phys. Commun. 189, 182 (2015).

[50] C. Duhr, T. Gehrmann, and M. Jaquier, J. High Energy Phys. 02 (2015) 077.

[51] P. Nogueira, J. Comput. Phys. 105, 279 (1993).

[52] J. A. M. Vermaseren, arXiv:math-ph/0010025.

[53] V. Shtabovenko, R. Mertig, and F. Orellana, Comput. Phys. Commun. 207, 432 (2016).

[54] F. Feng, Comput. Phys. Commun. 183, 2158 (2012).

[55] Z. Bern, L. J. Dixon, and D. A. Kosower, Nucl. Phys. B412, 751 (1994).

[56] T. Gehrmann and E. Remiddi, Nucl. Phys. B580, 485 (2000).

[57] J. M. Henn, Phys. Rev. Lett. 110, 251601 (2013).

[58] R. N. Lee, J. High Energy Phys. 04 (2015) 108.

[59] C. Meyer, J. High Energy Phys. 04 (2017) 006.

[60] O. Gituliar and V. Magerya, Comput. Phys. Commun. 219, 329 (2017).

[61] E. Remiddi and J. A. M. Vermaseren, Int. J. Mod. Phys. A 15, 725 (2000).

[62] S. Mantry and F. Petriello, Phys. Rev. D 81, 093007 (2010).

[63] M. Beneke and V. A. Smirnov, Nucl. Phys. B522, 321 (1998).
[64] C. Anastasiou, C. Duhr, F. Dulat, E. Furlan, T. Gehrmann, F. Herzog, and B. Mistlberger, J. High Energy Phys. 03 (2015) 091.

[65] C. Anastasiou, C. Duhr, F. Dulat, and B. Mistlberger, J. High Energy Phys. 07 (2013) 003.

[66] C. Anastasiou, C. Duhr, F. Dulat, E. Furlan, T. Gehrmann, F. Herzog, and B. Mistlberger, Phys. Lett. B 737, 325 (2014).

[67] Y. Li, A. von Manteuffel, R. M. Schabinger, and H. X. Zhu, Phys. Rev. D 91, 036008 (2015).

[68] Y. Li, A. von Manteuffel, R. M. Schabinger, and H. X. Zhu, Phys. Rev. D 90, 053006 (2014).

[69] S. Moch, J. A. M. Vermaseren, and A. Vogt, Nucl. Phys. B688, 101 (2004).

[70] A. Vogt, S. Moch, and J. A. M. Vermaseren, Nucl. Phys. B691, 129 (2004).

[71] See Supplemental Material at http://link.aps.org/ supplemental/10.1103/PhysRevLett.124.092001 for including Mathematica files Beamfunction.m, softfunction.m and TMDpdf.m, which includes Refs. [72-78].

[72] P. A. Baikov, K. G. Chetyrkin, and J. H. Kühn, Phys. Rev. Lett. 118, 082002 (2017).

[73] S. Moch, J. A. M. Vermaseren, and A. Vogt, Phys. Lett. B 625, 245 (2005).

[74] T. Gehrmann, E. W. N. Glover, T. Huber, N. Ikizlerli, and C. Studerus, J. High Energy Phys. 06 (2010) 094.

[75] T. Becher and M. Neubert, J. High Energy Phys. 06 (2009) 081; 11 (2013) 024(E).

[76] A. V. Manohar and I. W. Stewart, Phys. Rev. D 76, 074002 (2007).

[77] J. G. M. Gatheral, Phys. Lett. 133B, 90 (1983).

[78] J. Frenkel and J. C. Taylor, Nucl. Phys. B246, 231 (1984).

[79] A. A. Vladimirov, Phys. Rev. Lett. 118, 062001 (2017).

[80] G. Lustermans, W. J. Waalewijn, and L. Zeune, Phys. Lett. B 762, 447 (2016).

[81] G. Billis, M. A. Ebert, J. K. L. Michel, and F. J. Tackmann, arXiv:1909.00811.

[82] I. Balitsky and A. Tarasov, J. High Energy Phys. 10 (2015) 017.

[83] S. Marzani, Phys. Rev. D 93, 054047 (2016).

[84] I. Balitsky and A. Tarasov, J. High Energy Phys. 06 (2016) 164.

[85] B.-W. Xiao, F. Yuan, and J. Zhou, Nucl. Phys. B921, 104 (2017).

[86] D. Maitre, Comput. Phys. Commun. 174, 222 (2006).

[87] R. D. Ball et al. (NNPDF Collaboration), J. High Energy Phys. 04 (2015) 040.

[88] Yu. L. Dokshitzer, G. Marchesini, and G. P. Salam, Phys. Lett. B 634, 504 (2006).

[89] A. Mitov, S. Moch, and A. Vogt, Phys. Lett. B 638, 61 (2006).

[90] S. Moch and A. Vogt, Phys. Lett. B 659, 290 (2008).

[91] A. A. Almasy, S. Moch, and A. Vogt, Nucl. Phys. B854, 133 (2012).

[92] S. Catani and M. Grazzini, Phys. Rev. Lett. 98, 222002 (2007).

[93] I. W. Stewart, F. J. Tackmann, and W. J. Waalewijn, Phys. Rev. D 81, 094035 (2010).

[94] A. Behring, K. Melnikov, R. Rietkerk, L. Tancredi, and C. Wever, Phys. Rev. D 100, 114034 (2019). 
[95] R. Boughezal, C. Focke, X. Liu, and F. Petriello, Phys. Rev. Lett. 115, 062002 (2015).

[96] J. Gaunt, M. Stahlhofen, F. J. Tackmann, and J. R. Walsh, J. High Energy Phys. 09 (2015) 058.

[97] R. Kelley, M. D. Schwartz, R. M. Schabinger, and H. X. Zhu, Phys. Rev. D 84, 045022 (2011).

[98] P. F. Monni, T. Gehrmann, and G. Luisoni, J. High Energy Phys. 08 (2011) 010.

[99] I. Moult, G. Vita, and K. Yan, arXiv:1912.02188.

[100] L. Cieri, C. Oleari, and M. Rocco, Eur. Phys. J. C 79, 852 (2019).

[101] L. Buonocore, M. Grazzini, and F. Tramontano, arXiv: 1911.10166.

[102] R. Angeles-Martinez, M. Czakon, and S. Sapeta, J. High Energy Phys. 10 (2018) 201.
[103] S. Catani, S. Devoto, M. Grazzini, S. Kallweit, J. Mazzitelli, and H. Sargsyan, Phys. Rev. D 99, 051501(R) (2019).

[104] A. Hornig, C. Lee, I. W. Stewart, J. R. Walsh, and S. Zuberi, J. High Energy Phys. 08 (2011) 054; 10 (2017) 101(E).

[105] A. J. Larkoski, I. Moult, and B. Nachman, arXiv: 1709.04464.

[106] M. A. Ebert, I. W. Stewart, and Y. Zhao, Phys. Rev. D 99, 034505 (2019).

[107] M. A. Ebert, I. W. Stewart, and Y. Zhao, J. High Energy Phys. 09 (2019) 037.

[108] M. A. Ebert, I. W. Stewart, and Y. Zhao, arXiv: 1910.08569.

[109] X. Ji, Y. Liu, and Y.-S. Liu, arXiv:1910.11415.

[110] X. Ji, Y. Liu, and Y.-S. Liu, arXiv:1911.03840. 